\title{
MicroRNA-375 regulates proliferation and apoptosis of glioma cancer cells by inhibiting CTGF-EGFR signaling pathway
}

\author{
Zhang $\mathrm{LX}^{1,3}$, Jin $\mathrm{W}^{2}$, Zheng $\mathrm{J}^{3}$, Dai $\mathrm{YX}^{2}$, Song $\mathrm{Y}^{1}$, Ni $\mathrm{HB}^{2}$, Jiang $\mathrm{J}^{2}$, Liang $\mathrm{WB}^{1,2}$ \\ Department of Neurosurgery, Nanjing Drum Tower Hospital Clinical College of Nanjing \\ Medical University, Jingsu, China. liangweibang0925@126.com
}

\begin{abstract}
AIM: To evaluate the correlation between miRNA-375 and cell proliferation and apoptosis in glioma cancer cell. METHODS: Collecting 30 cases of glioma cancer patients and 30 cases of cerebral infarction patients. The miRNA-375 and CTGF protein expressions were evaluated by ISH and IHC methods. In the cell experiment, the U87 cells were divided into 3 groups: NC group (the cells were treated with normal method); BL group (the cells were transfected with empty vector) and miRNA group (the cells were transfected with miRNA-375). The U87 cell proliferation and apoptosis rates and cell cycle of the different groups were measured by MTT and flow cytometry. The relative proteins (CTGF, EGFR, AKT, Erk and P21) expressions were measured by WB assay. RESULTS: The miRNA-375 and CTGF expressions of glioma cancer tissues were significantly different compared with those of no-cancer tissues ( $p<0.05$, respectively). In the cell experiments, the cell proliferation of miRNA group was significantly decreased compared with that of NC group $(p<0.05)$; the cell apoptosis and G1 phase rate of miRNA group was significantly decreased compared with NC group ( $p<0.05$, respectively). Depending on the WB assay, the CTGF, EGFR, AKT, Erk and P21 proteins expressions of miRNA group were significantly different compared with proteins expressions of NC group ( $p<0.05$, respectively).

CONCLUSION: miRNA-375 over-expression suppresses glioma cancer cells development via CTGF-EGFR pathway (Fig. 3, Ref. 30). Text in PDF www.elis.sk.

KEY WORDS: miRNA-375, CTGF, EGFR, glioma cancer.
\end{abstract}

\section{Introduction}

Glioma is the most common primary central nervous system tumor. 50 60\% of malignant brain tumors are gliomas (1). The current treatment strategies for glioma are surgery, radiation therapy, chemotherapy, or other adjuvant therapy (2-4). However, the high metastasis rate and the tolerance to radiotherapy and chemotherapy make the prognosis of glioma very poor (5-7). Intervention or licensing of disease from a molecular perspective provides new insights into the current treatment dilemma. microRNA (miRNA) miRNA plays an important regulatory role in tumorigenesis and development, and plays a regulatory role in the proliferation, invasion and apoptosis of cancer (8-10). In some previous studies it was shown that miRNA-375 had anti-tumor effects on some kinds of cancer (11-14). However, it has been unclear that there is a correlation between miRNA-375 and glioma. In our present study, we firstly evaluated the miRNA-375 in the normal and glioma cancer tissues, and then we assessed miRNA-375 over-expression in glioma cancer cell biological activity.

${ }^{1}$ Department of Neurosurgery, Nanjing Drum Tower Hospital Clinical College of Nanjing Medical University, Nanjng, Jingsu, China, ${ }^{2}$ Department of Neurosurgery, The Affiliated Drum Tower Hospital, School of Medicine, Nanjing University, Nanjing, Jingsu, China, and ${ }^{3}$ Department of Neurosurgery, Suqian People's Hospital, Nanjing Drum Tower Hospital Group, Suqian, Jingsu, China

Address for correspondence: W.B. Liang, Department of Neurosurgery, Nanjing Drum Tower Hospital Clinical College of Nanjing Medical University, Nanjng 210008, Jingsu, China.

\section{Materials and Methods}

\section{Clinical data}

The glioma cancer tissues were collected from 30 cases of glioma cancer patients who were treated in our hospital, and the normal control tissues were collected from 30 cases of cerebral infarction patients who were treated at the same periods. The tissues were fixed in $4 \%$ paraformaldehyde, Paraffin embedded section as $4 \mu \mathrm{m}$.

\section{In situ hybridization assay}

Paraffin wax after dewaxing, Placed in a mixture of $0.8 \%$ pepsin and hydrochloric acid, digest for $10 \mathrm{~min}$ in water bath at $37^{\circ} \mathrm{C}$, Using TBS to wash $5 \mathrm{~min}$, dehydrate ethanol, and then dry at room temperature. Denaturation at $98{ }^{\circ} \mathrm{C}$ for $10 \mathrm{~min}$, Ice bath annealing, hybridization for $1 \mathrm{~h}$ at the $37^{\circ} \mathrm{C}$ in the water-bath. Washing by TBS $5 \min \times 3$ times, after incubation with alkaline phosphatase labeled digoxin antibody, incubated at room temperature for $0.5 \mathrm{~h}$, after washing for 2 times, adding BCIP/NBT and color in the dark, $0.3 \%$ Nuclearfast red lining dye, dehydrated, transparent after mounting.

\section{Immunohistochemistry (IHC)O}

Monoclonal antibody CTGF SP kit was purchased from Sigma (1 : 100). Using $3 \% \mathrm{H}_{2} \mathrm{O}_{2}$ to block endogenous peroxidase, microwave heating antigen repair, $10 \%$ normal goat serum was incubated with primary antibody at $-4{ }^{\circ} \mathrm{C}$ overnight in the refrig- 

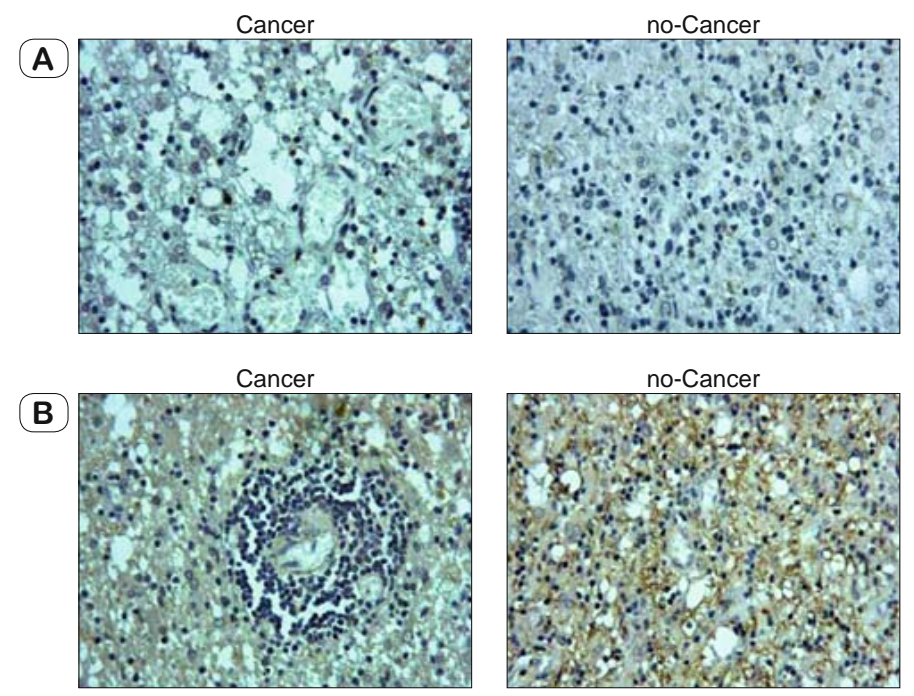

Fig. 1. Clinical data. A. The CTGF protein expression of different tissues by IHC $(\times 200), * * * \mathbf{p}<0.05$, compared with cancer tissues, B. The miRNA-375 expression of different tissues by ISH $(\times 200),{ }^{* * *} \mathrm{p}<0.05$, compared with cancer tissues.

erator. Sections were washed by PBS, after that, adding second and third anti-body, DAB color, hematoxylin staining, conventional mounting.

\section{Cell culture and grouping}

Human glioma cells U87 were cultured by RPMI-1640 with $10 \%$ fetal bovine serum (FBS) in the incubator $\left(37^{\circ} \mathrm{C}, 5 \% \mathrm{CO}_{2}\right)$, Digested with $0.25 \%$ trypsin, the passage was changed every 2 days. The U87 cells were divided into 3 groups: NC group (the cells were treated with normal methods); BL group (the cells were transfected with empty vector) and miRNA group (the cells were transfected with miRNA-375). After transfection for $48 \mathrm{~h}$, experiments were started.

\section{MTT assay}

The cells of different groups were inoculated in 96-holes as $1 \times 10^{6} /$ hole. $20 \mu \mathrm{l} \mathrm{MTT}(5 \mathrm{~g} / \mathrm{L})$ was added to every hole and continued to be cultured for $4 \mathrm{~h}$, the culture medium was added and DMSO 1501 was added. The absorbance was detected at $490 \mathrm{~nm}$ with an enzyme analyzer. The cell proliferation rates of different groups were measured.

\section{The cell apoptosis by flow cytometry}

The cells were inoculated in the 6 -hole plate as $1 \times 10^{5} /$ hole. A single cell suspension was made by trypsin digestion, and PBS was used to wash 3 times. According to the instructions, the cells were stained with PI/Annexin V, and the apoptosis rate of each group was detected by light staining $20 \mathrm{~min}$. The experiment was repeated 3 times, with 3 holes at each time.

\section{The cell cycle by flow cytometry}

The cells were inoculated in the 6-hole plate as $1 \times 10^{6} /$ hole. A single cell suspension was made by trypsin digestion, and PBS was used to wash 3 times. After adding $1 \mathrm{~mL}$ PBS solution, the cells were suspended and then $2 \mathrm{~mL} 70 \%$ ethanol were slowly dropped in $\left(-20{ }^{\circ} \mathrm{C}\right.$ pre-cooling $)$. After mixing, they were fixed overnight at $4{ }^{\circ} \mathrm{C}$. Centrifuged at $1000 \mathrm{r} / \mathrm{min}$ for $1.5 \mathrm{~min}, 70 \%$ ethanol solution was discarded, then it was washed 2 times at 4 ${ }^{\circ} \mathrm{C}$ with PBS solution, then the supernatant was discarded. $800 \mu \mathrm{l}$ of $20 \mu \mathrm{g} / \mathrm{mL}$ PI solution was added containing $200 \mu \mathrm{g} / \mathrm{mL}$ RNase, mixed, cultured at $37^{\circ} \mathrm{C}$ for $30 \mathrm{~min}$ in dark, after that Machine inspection was done.

\section{Western Blot assay}

The cells were collected from different groups, protein concentration was measured by BCA method according to the operation of the nucleoprotein extraction kit, $12 \%$ polyacrylamide gel and $5 \%$ concentrated gum were prepared, and the sample was 30 $\mu \mathrm{g}$, after the vertical electrophoresis, the gel was transferred to the PVDF film by wet process. According to the protein marker cut
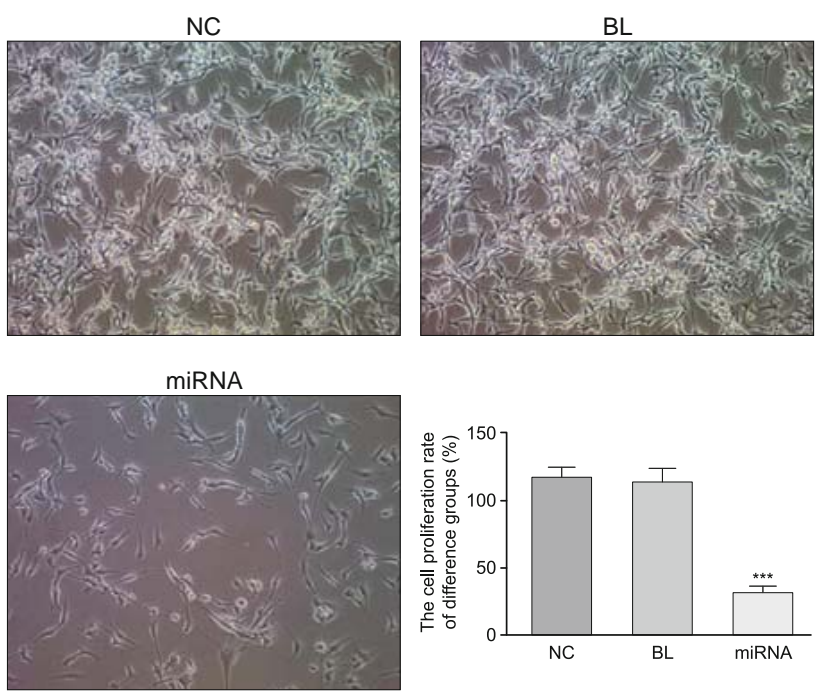

Fig. 2. The cell proliferation rate of different groups. $* * * p<0.05$, compared with NC group. 
film, ir was washed with TBST solution, with $5 \%$ skimmed milk powder on the shaking table atroom temperature closed for $1.5 \mathrm{~h}$, and then wash away with skim milk powder solution. Adding the primary antibodies CTGF, EGFR, AKT, Erk, P21 and GAPDH (1 $: 1000)$ to culture, The PVDF membrane and the corresponding antibody were placed in the incubation bag and incubated at $4{ }^{\circ} \mathrm{C}$ over-night. Next day, the PVDF membranes were washed by TBST (10 min x 3times). Adding goat anti mouse HRP-IgG as $1: 5000$ to culture for $1.5 \mathrm{~h}$, after washing by TBST, ECL light treatment was carried out to take pictures and save the results.

\section{Statistical analysis}

All experiments were performed three times and data were analyzed with GraphPad Prism 5. Statistical evaluation of data was performed using the t-test. $\mathrm{p}<0.05$ was considered to be statistically significant. Spearman's nonparametric correlation test was performed to test the correlation between the expression levels of miR-181b and IGF-1R by SPSS 19.0 software.

\section{Results}

\section{Clinical data}

Compared with Cancer tissues, the CTGF protein expressions of non-Cancer tissues were significantly down-regulated and the miRNA-375 expressions of non-Cancer tissues were significantly up-regulated ( $p<0.05$, respectively). The relative data are shown in Figure 1. Depending on these results, we inferred that miRNA-375 might be in negative correlation with CTGF in cancer tissues.

miRNA-375 suppresses cell proliferation in vitro

With miRNA-375 infection, the cell proliferation rate of miRNA was significantly suppressed compared with that of NC group
A
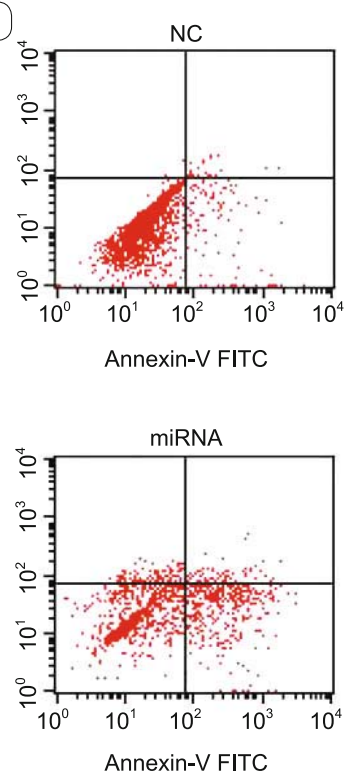

(C)

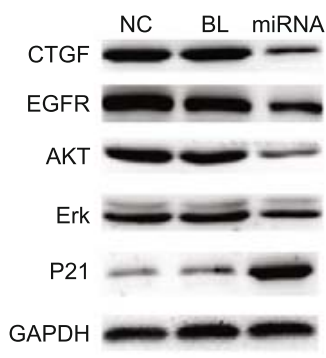

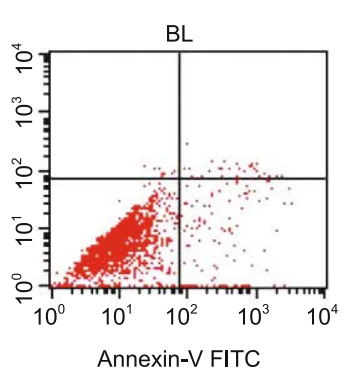
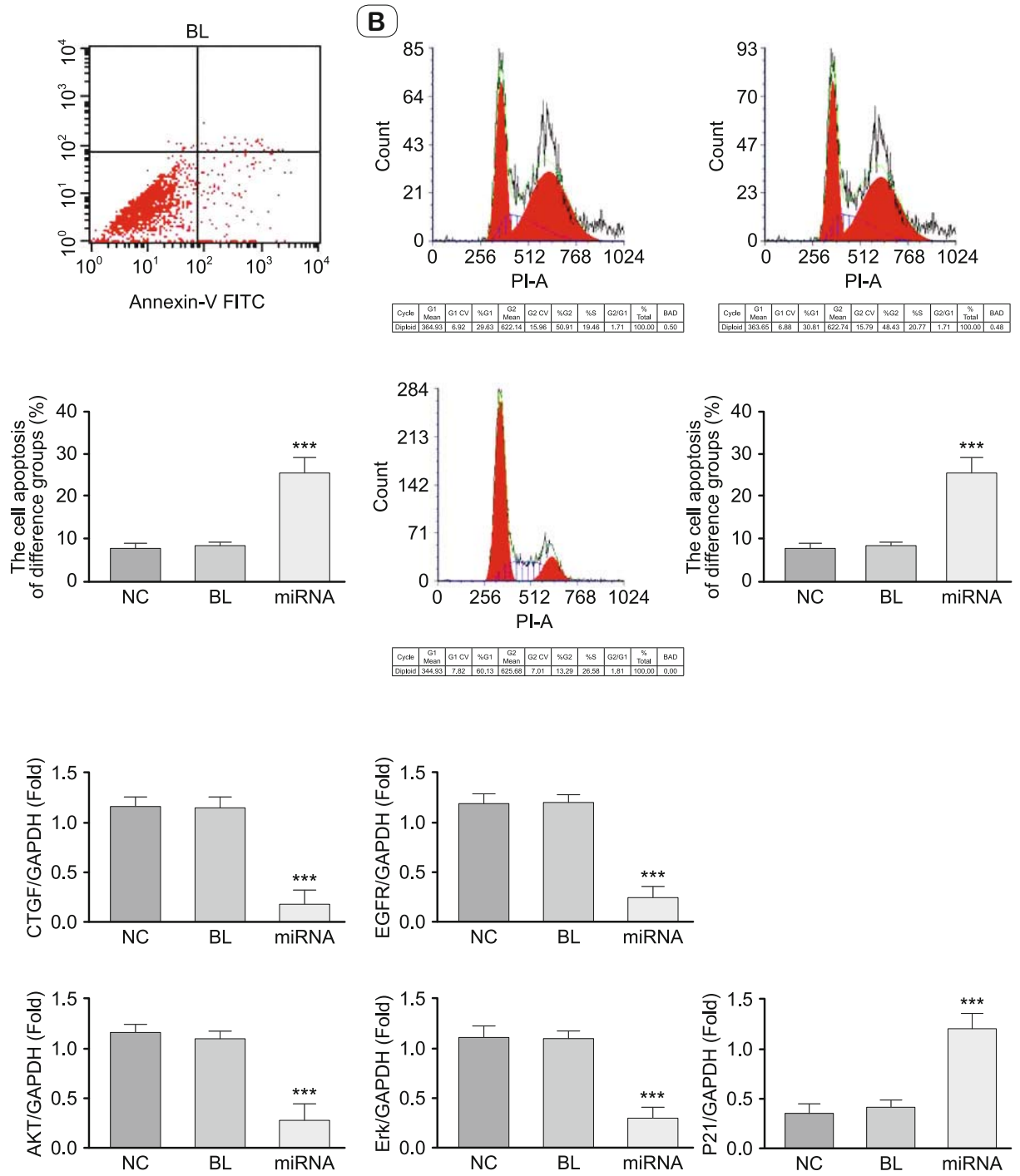

Fig. 3. The cell experiments in U87 cell. A. The cell proliferation of different groups by MTT, $* * * \mathbf{p}<0.05$, compared with NC group, B. The G1 phase rate of different groups, $* * * \mathbf{p}<0.05$, compared with NC group, C. The relative proteins expression of different groups by WB assay, ${ }^{* * *} \mathrm{p}<0.05$, compared with NC group. 
$(\mathrm{p}<0.05)$, however, there were no significant differences between $\mathrm{NC}$ and BL groups that shows that the empty vector had no effects on cell proliferation $(\mathrm{p}<0.05)$. The relative data are shown in Figure 2.

\section{miRNA-375 stimulated cell apoptosis rate}

The data showed that the cell apoptosis of miRNA group was significantly up-regulated compared with $\mathrm{NC}$ group $(\mathrm{p}<0.05)$; however, there were significant differences in cell apoptosis rates of $\mathrm{NC}$ and $\mathrm{BL}$ groups $(\mathrm{p}<0.05)$. The relative data are shown in Figure $3 \mathrm{~A}$.

\section{miRNA-375 up-regulates G1 phase in cell cycle}

The G1 phase of miRNA group was significantly enhanced compared with NC group $(\mathrm{p}<0.05)$; however, there were no significant differences between $\mathrm{G} 1$ phase of $\mathrm{NC}$ and $\mathrm{BL}$ groups $(\mathrm{p}>$ $0.05)$. The relative data are shown in Figure $3 \mathrm{~B}$.

\section{miRNA-375 has effects to relative proteins}

Compared with NC group, the CTGF, EGFR, AKT and Erk proteins expressions of miRNA group were significantly down-regulated ( $\mathrm{p}<0.05$, respectively), P21 protein expression of miRNA group was up-regulated $(p<0.05)$. However, there were no significant differences between NC and BL groups in CTGF, EGFR, AKT, Erk and P214 proteins expressions ( $\mathrm{p}>0.05$, respectively). The relative data are shown in Figure $3 \mathrm{C}$.

\section{Discussion}

At present, due to the unlimited proliferation of malignant tumor cells, tumor patients are mostly incurable, have low survival rate and low quality of life, and so on, so that it has become the focus of attention world-wide. It is very important to study the mechanism of the occurrence and development of malignant tumor for its early diagnosis and targeted therapy. Connective tissue growth factor (CTGF) expression was closely correlated with the development of cancer and was involved in cell proliferation, development, adhesion, migration, angiogenesis, and predicts prognosis (15-20). miRNA regulates mRNA levels by targeting 3'UTR of the target molecule, thereby regulating protein levels (21, 22). Previous study found that miRNA-375 had anti-tumor effects targeted by CTGF (23). However, it has the miRNA-375 expression in the glioma and the correlation between miRNA-375 and CTGF in the glioma cancer has been unclear. In our present study, depending on the clinical data, we have found that miRNA-375 had low expression and CTGF protein had high expression in the glioma tissues. Based on these results, we inferred that miRNA-375 might target CTGF in glioma. In the subsequent experiments we wanted to explain the effects and mechanism of miRNA-375 in the glioma cancer cells.

In our present study, the results have shown that miRNA-375 over-expression can suppress glioma cancer cell U87 cell proliferation and stimulate cell apoptosis by staining the cell cycle in G1 phase. In order to explain the mechanism of miRNA-375 in the development of glioma cancer cell, we evaluated the relative proteins expression at the molecular biological level.
Epidermal growth factor receptor (EGFR), a membrane receptor with tyrosine kinase activity, is expressed ubiquitously in human epidermal and stromal cells and is highly expressed in a variety of human malignancies (24). EGFR has an important role of downstream gene of CTGF $(25,26)$. The signal transduction effects mediated by EGFR are multi directional, including proliferation, migration, cell differentiation and the stability of the internal environment, and are closely related to cell regeneration and the occurrence and development of malignant tumors $(27,28)$. EGFR also mediated the AKT/Erk/P21 pathway, AKT/Erk/P21 signaling pathway has a pivotal role in cell apoptosis and in the cell development $(29,30)$. That pathway regulates cell apoptosis via controlling the cell cycle. In this study, we found that the AKT and Erk protein expressions were significantly suppressed and P21 protein expression was significantly stimulated in miRNA-375 over-expression group. Depending on these results, we inferred that miRNA-375 may suppress glioma cancer cell U87 cell proliferation via AKT/Erk/ P21 signaling pathway.

In conclusion, miRNA-375 has anti-tumor effects via CTGF/ EGFR/AKT/Erk/P21 pathway in U87 cell, a type of glioma cancer cell lines of in vitro study.

\section{References}

1. Shipman L. Glioma: Tumour cell teamwork. Nat Rev Cancer 2016; 16 (1): 2 .

2. Furnari FB, Fenton T, Bachoo RM et al. Malignant astrocytic glioma: genetics, biology, and paths to treatment. Genes Dev 2007; 21 (21): 2683-2710

3. Zhao B, Bian EB, Li J et al. New advances of microRNAs in glioma stem cells, with special emphasis on aberrant methylation of microRNAs. J Cell Physiol 2014; 229 (9): 1141-1147.

4. Sabbagh AJ, Alaqeel AM. Focal brainstem gliomas. Advances in intraoperative management. Neurosciences (Riyadh) 2015; 20 (2): 98-106.

5. Castro MG, Lowenstein PR. Neuro-oncology: The long and winding road-gene therapy for glioma. Nat Rev Neurol 2013, 9 (11): 609-610.

6. Hashizume R, Andor N, Ihara Y et al. Pharmacologic inhibition of histone demethylation as a therapy for pediatric brainstem glioma. Nat Med 2014; 20 (12): 1394-1396.

7. Reardon DA, Wen PY. Glioma in 2014: unraveling tumour heterogeneity-implications for therapy. Nat Rev Clin Oncol 2015; 12 (2): 69-70.

8. Urbano J, Cabrera M, Alonso-Burgos A. Sclerosis and varicocele embolization with N-butyl cyanoacrylate: experience in 41 patients. Acta Radiol 2014; 55 (2): 179-185.

9. Romero-Cordoba SL, Salido-Guadarrama I, Rodriguez-Dorantes M et al. miRNA biogenesis: biological impact in the development of cancer. Cancer Biol Ther 2014; 15 (11): 1444-1455.

10. Shin VY, Chu KM. MiRNA as potential biomarkers and therapeutic targets for gastric cancer. World J Gastroenterol 2014; 20 (30): 1043210439.

11. Szczyrba J, Nolte E, Wach S et al. Downregulation of Sec23A protein by miRNA-375 in prostate carcinoma. Mol Cancer Res 2011; 9 (6): 791-800. 
12. Zhou N, Qu Y, Xu C et al. Upregulation of microRNA-375 increases the cisplatin-sensitivity of human gastric cancer cells by regulating ERBB2. Exp Ther Med 2016; 11 (2): 625-630.

13. Tsukamoto Y, Nakada C, Noguchi T et al. MicroRNA-375 is downregulated in gastric carcinomas and regulates cell survival by targeting PDK1 and 14-3-3zeta. Cancer Res 2010; 70 (6): 2339-2349.

14. Cheng L, Zhan B, Luo P et al. miRNA-375 regulates the cell survival and apoptosis of human non-small cell carcinoma by targeting HER2. Mol Med Rep 2017; 15 (3): 1387-1392.

15. Ding S, Duan H, Fang F et al. CTGF promotes articular damage by increased proliferation of fibroblast-like synoviocytes in rheumatoid arthritis. Scand J Rheumatol 2016; 45 (4): 282-287.

16. Shi C, Li G, Tong Y et al. Role of CTGF gene promoter methylation in the development of hepatic fibrosis. Am J Transl Res 2016; 8 (1): 125-132.

17. Hendesi H, Barbe MF, Safadi FF et al. Integrin mediated adhesion of osteoblasts to connective tissue growth factor $(\mathrm{CTGF} / \mathrm{CCN} 2)$ induces cytoskeleton reorganization and cell differentiation. PLoS One 2015; 10 (2): e0115325.

18. Han Q, Zhang HY, Zhong BL et al. MicroRNA-145 Inhibits Cell Migration and Invasion and Regulates Epithelial-Mesenchymal Transition (EMT) by Targeting Connective Tissue Growth Factor (CTGF) in Esophageal Squamous Cell Carcinoma. Med Sci Monit 2016; 22: 3925-3934.

19. Gahr S, Mayr C, Kiesslich T et al. The pan-deacetylase inhibitor panobinostat affects angiogenesis in hepatocellular carcinoma models via modulation of CTGF expression. Int J Oncol 2015; 47 (3): 963-970.

20. Yue Han, Yuxin Chen. MiR-24 may act as an oncomiR in human astrocytoma by targeting CDKN1B/p27. Adv Bio Med 2016; 1 (1): 41-54.

21. Iorio MV, Croce CM. MicroRNA dysregulation in cancer: diagnostics, monitoring and therapeutics. A comprehensive review. EMBO Mol Med 2017; 9 (6): 852.
22. Wang C, Ji B, Cheng B, Chen J. Neuroprotection of microRNA in neurological disorders (Review). Biomed Rep 2014; 2 (5): 611-619.

23. Alam KJ, Mo JS, Han SH et al. MicroRNA 375 regulates proliferation and migration of colon cancer cells by suppressing the CTGF-EGFR signaling pathway. Int J Cancer 2017; 141 (8): 1614-1629.

24. Hirano T, Yasuda $\mathbf{H}$, Tani $\mathbf{T}$ et al. In vitro modeling to determine mutation specificity of EGFR tyrosine kinase inhibitors against clinically relevant EGFR mutants in non-small-cell lung cancer. Oncotarget 2015; 6 (36): 38789-803.

25. Kidd M, Schimmack S, Lawrence B et al. EGFR/TGF $\alpha$ and TGF $\beta$ / CTGF Signaling in Neuroendocrine Neoplasia: Theoretical Therapeutic Targets. Neuroendocrinol 2013; 97 (1): 35-44.

26. Bundela S, Sharma A, Bisen PS. Potential therapeutic targets for oral cancer: ADM, TP53, EGFR, LYN, CTLA4, SKIL, CTGF, CD70. PLoS One 2014; 9 (7): e102610.

27. Fan JB, Liu W, Yuan K et al. EGFR trans-activation mediates pleiotrophin-induced activation of Akt and Erk in cultured osteoblasts. Biochem Biophys Res Commun 2014; 447 (3): 425-430.

28. Lv S, Dai C, Liu Y, Cell surface protein C23 affects EGF-EGFR induced activation of ERK and PI3K-AKT pathways. J Mol Neurosci 2015; 55 (2): 519-524.

29. Tyagi N, Bhardwaj A, Singh AP et al. p-21 activated kinase 4 promotes proliferation and survival of pancreatic cancer cells through AKTand ERK-dependent activation of NF- $\mathrm{\kappa B}$ pathway. Oncotarget 2014; 5 (18): 8778-8789.

30. Liu Y, Bi T, Wang G et al. Lupeol inhibits proliferation and induces apoptosis of human pancreatic cancer PCNA-1 cells through AKT/ ERK pathways. Naunyn Schmiedebergs Arch Pharmacol 2015; 388 (3): 295-304.

Received September 25, 2017. Accepted October 20, 2017. 